\title{
Ultrafast photoresponses of CdS nanoparticles in self-assembled films
}

\author{
Hiroshi Inoue ${ }^{1}$, Robert S. Urquhart ${ }^{2}$, Toshihiko Nagamura ${ }^{1,3}$, Franz Grieser", \\ Hiroshi Sakaguchi ${ }^{3}$, and D. Neil Furlong ${ }^{2}$
}

${ }^{1}$ Department of Electronic Materials Science, Graduate School of Electronic Science and Technology, Shizuoka University, 3-5-1 Johoku, Hamamatsu 432-8011, Japan.

${ }^{2}$ CSIRO, Division of Chemicals and Polymers, Private Bag 10, Rosebank MDC, Clayton, Victoria 3169, Australia.

${ }^{3}$ Crystalline Films Laboratory, Research Institute of Electronics, Shizuoka University, 3-5-1 Johoku, Hamamatsu 432-8011, Japan.

${ }^{4}$ Advanced Mineral Products Research Centre, School of Chemistry, University of Melbourne, Parkville, Victoria 3052, Australia.

\begin{abstract}
The ultra-fast dynamics of transient photobleaching and recovery of cadmium sulphide nanoparticles with sizes of about $4.0 \mathrm{~nm}$ diameter in self-assembled films of ethylenediamine amphiphiles was studied upon excitation with a femtosecond laser at $\mathbf{4 0 0}$ nm. Maximum photobleaching occurred within about 1 picosecond. The bleaching then recovered with multi-exponential kinetics. The peak of the transient photobleaching spectrum showed a progressive red shift as the time between the pump and probe pulses was increased. The observed transient spectral shifts have been explained in terms of the number of trapping sites present on the particle surface.
\end{abstract}

Keywords : nanoparticles, semiconductor, self-assembled films, transient photobleaching, femtosecond laser

\section{Introduction}

During the past twenty years, researches on the formation and properties of "ultrasmallparticles" in particular nanoparticles, has become increasingly popular in various fields of chemistry and physics. One specific area of research dealing with the recombination process of electron-hole pairs in semiconductor nanoparticles has grown significantly during the past ten years. [1-4] Electronic excitation of a semiconductor nanoparticle generates a loosely bound electron-hole pair (the Wannier exciton), usually delocalized over a length much longer than the lattice constant. As the diameter of the semiconductor nanoparticle approaches this exciton diameter, the optical bandgap or exciton energy increases, which results in a blue shift of the absorption spectra-the quantum-size effect. For CdS, the exciton diameter is 5-6 nm.[1] Several methods have been reported for the preparation of nanoparticles. A large percentage of atoms making up the nanoparticles exist on or near the particle surface, which act as electron and/or hole traps upon optical excitation. Efficient transient bleaching has been observed for semiconductor nanoparticles by several groups [1-3]. Based on the picosecond and nanosecond pump-probe results, Wang et al. suggested that surface trapped electron-hole (eh) pairs, through interaction with the exciton, are responsible for the observed transient bleaching for the quantum-confined CdS particles in polymer films [1,2]. A short pulse laser excitation initially generates a bound exciton. This bound exciton is rapidly ( $<1$ ps) trapped by the surface, and is known to subsequently decay with double-exponential kinetics.

In this paper, we report on the ultrafast responses of CdS nanoparticles in self-assembled films using femtosecond laser flash photolysis. 
Results from the present femtosecond laser study reveal that the recovery of transient photobleaching behavior in nanoparticles within a self-assembled matrix follows complex kinetics due to the existence of a distribution of particle sizes and a variety of surface traps on the nanoparticles.

\section{Method}

2.1. Preparation of self-assembled cast film Ditetradecyl-N-[4-[[6-(N,N',N'-trimethylethylenediamino)-hexyl]oxy]benzoyl]-Lglutamate (DTG) was a gift from Dr. Izumi Ichinose (Kyushu University, Japan). The structure of DTG is shown in Fig. 1. Cast films of the cadmium salt of DTG, [Cd(DTG)2], were prepared following a procedure similar to that outlined by Ichinose et al.[5] Suspensions containing the monohydrochloride of DTG were formed by adding appropriate amounts of DTG, aqueous $0.1 \mathrm{M} \mathrm{HCl}$ and water. After addition of the components, the surfactant was dispersed using a Heat Systems-Ultrasonics Sonicator (Model W-220F). The system was sonicated until it was clear enough to see through. The resulting supernatant was used in all further experiments. Suspensions containing $0.018 \mathrm{M}$ Cd(DTG)2 were prepared immediately prior to film formation by adding $172 \mathrm{ml}$ of an aqueous solution of $0.259 \mathrm{M}$ cadmium chloride to $2.3 \mathrm{ml}$ of the supernatant (this yielded a DTG: $\mathrm{Cd}^{2+}$ molar ratio of $2: 1$ ). One $\mathrm{ml}$ of the $\mathrm{Cd}(\mathrm{DTG})_{2}$ solution was added dropwise to a square frame made out of a black and white negative film which was placed on a Telfon base. Films of $\mathrm{Cd}(\mathrm{DTG})_{2}$ were then placed in a laminar flow hood for two days to allow the solvent to evaporate. The $\mathrm{Cd}(\mathrm{DTG})_{2}$ film which was exposed to dry $\mathrm{H}_{2} \mathrm{~S}$ was stored in a vacuum desiccator (pressure $=7 \mathrm{kPa}$ ) after film formation. The absorption and fluorescence spectra are shown in Fig. 2. The broad and featureless absorption spectrum indicates that there is some variation in the size of the CdS nanoparticles in the self-assembled film. The bandgap of bulk CdS is $2.42 \mathrm{eV}$ (i.e. $510 \mathrm{~nm}$ ) and shifts to higher energies as the size of the particles becomes smaller due to the quantumsize effects. In the case of our sample, the absorption onset wavelength is much less than $500 \mathrm{~nm}$. This behavior clearly indicates that the

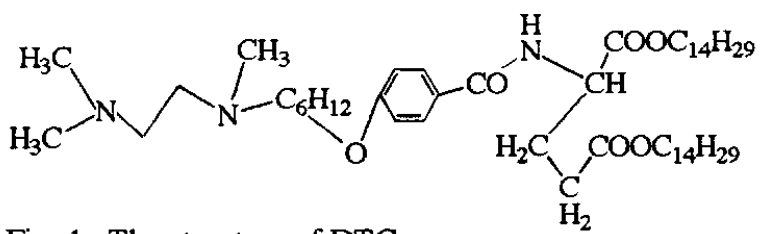

Fig. 1. The structure of DTG.

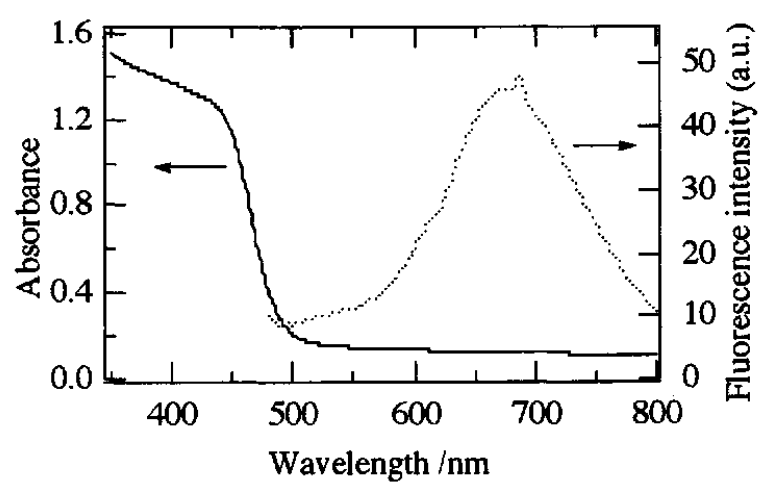

Fig. 2. UV-visible (full line) absorption and fluorescence (dashed) spectra of the CdS nanoparticles in self-assembled film of DTG.

size of the particles is in the quantum-size regime. The size of CdS particles in the film was estimated using the absorption spectra shown in Fig. 2 and the calibration curve of Henglein et al.[6] The average diameter of CdS nanoparticles in DTG film was estimated at 4.0 nm. The fluorescence spectrum exhibits a broad band in the range of $500-800 \mathrm{~nm}$. This broad emission is due to the surface states commonly observed for ultrasmall CdS particles. It seems reasonable to suppose that a large fraction of electrons-holes recombination will follow a path via surface states.

2.2. Femtosecond transient bleaching measurements.

Transient bleaching measurements were performed using a pump-probe method with a regeneratively amplified, mode-locked fs $\mathrm{Ti}$ sapphire laser.[3] A schematic diagram of the experimental set-up is shown in Fig. 3. The amplified Ti-sapphire laser delivered pulses with a full width at half maximum (FWHM) of 200-250 fs at a $10 \mathrm{~Hz}$ repetition rate and had a maximum power output of $6 \mathrm{~mJ} /$ pulse at 800 $\mathrm{nm}$. The amplified output of the laser was frequency doubled by a $\beta-\mathrm{BaB}_{2} \mathrm{O}_{4}$ (BBO) crystal to generate $400 \mathrm{~nm}$ light, which was used as the pump source $(40 \mu \mathrm{J} /$ pulse $)$. The remaining fundamental light was focused onto a 
$1 \mathrm{~cm}$ cell containing a $2: 1 \mathrm{v} / \mathrm{v}$ mixture of $\mathrm{D}_{2} \mathrm{O} / \mathrm{H}_{2} \mathrm{O}$ to generate a white light continuum probe. The transient bleaching and decay dynamics were measured by a dual photodiode array system (Hamamatsu Photonics C6140PMA) using an optical delay ( $2 \mu \mathrm{m} / \mathrm{step})$. The intensities of the white probe light, with and without the pump pulse, were averaged 20 times. Kinetics were analyzed by the least squares method.

\section{Results and discussion}

We demonstrated previously, from femtosecond photobleaching experiments, the ultrafast photoresponses of CdS nanoparticles in Nafion films originates from the bleaching of the excitonic band absorption.[3] Fig. 4 shows the time-resolved transient photobleaching spectra at different times after excitation with $400 \mathrm{~nm}$ laser pulses. A marked decrease in the absorbance of the particles was observed within 1 picosecond (ps) after the excitation pulses. The maximum of transient photobleaching spectrum occurs at about $443 \mathrm{~nm}(=2.80 \mathrm{eV})$ and it should correspond to the exciton peak, lowest energy transition band.[7] The bleaching peak shifted from a higher to a lower energy region during the recovery process as shown in Fig. 4. Kamalov et al. have observed similar phenomena and proposed that the shift in the peak of the bleaching band is due to a lower energy trap distribution on the particle. [8] Another possible explanation is such that the bleaching shift is caused by the size distribution of CdS nanoparticles by the mechanism that excitons or electron-hole pairs decay faster in a smaller particle than in a larger one.[3] We have confirmed that this mechanism is also predominant in the present case because no rise was observed in the time profile of transient bleaching recovery in the longer wavelength region as mentioned below (see below).

Fig. 5 shows the time profiles of transient bleaching probed at 440 and $470 \mathrm{~nm}$ after excitation with $400 \mathrm{~nm}$ pump pulses. Maximum bleaching occurred within about $1 \mathrm{ps}$. As shown in Fig. 5(a), a double exponential fit to the recovery of the bleaching yielded a fast component ( $\tau_{1}=15 \mathrm{ps}, f_{1}=37 \%$ ), a slow one ( $\left.\tau_{2}=159 \mathrm{ps}, f_{2}=40 \%\right)$ and a very long-lived one $\left(\tau_{3}>1 \mathrm{~ns}, f_{3}=23 \%\right)$. The rapid recovery is

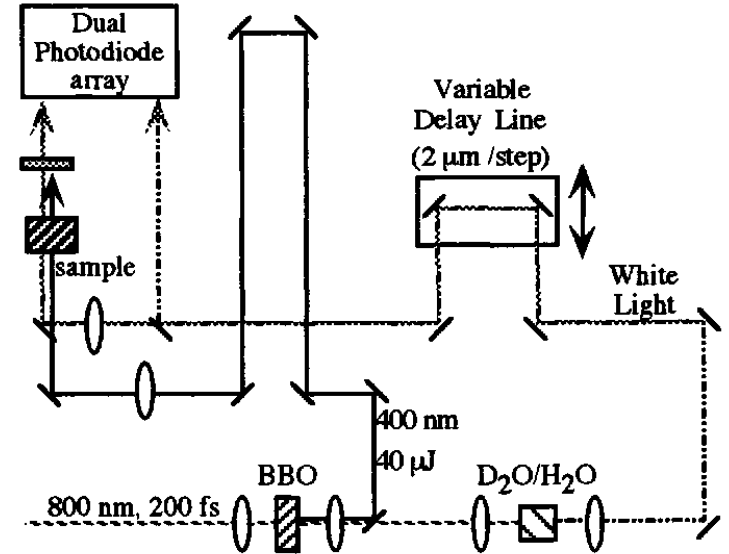

Fig. 3. Schematic diagram of the experimental setup used in the femtosecond transient bleaching measurements.

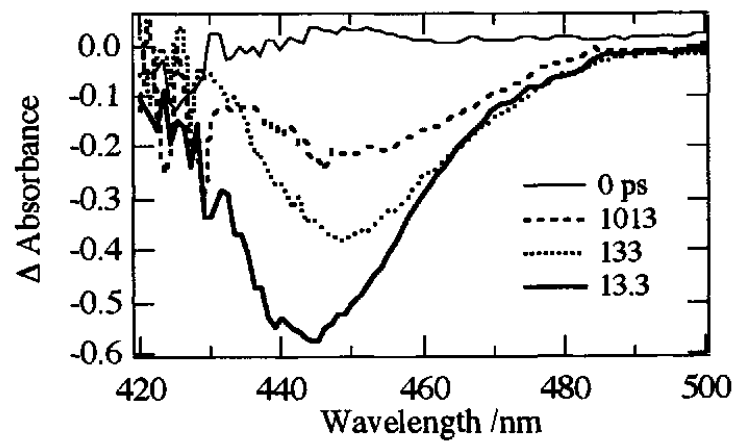

Fig. 4. Time-resolved bleaching spectra of $\mathrm{CdS}$ nanoparticles in DTG film obtained at different times. Pump energy $=40 \mu \mathrm{J}$ per pulse.

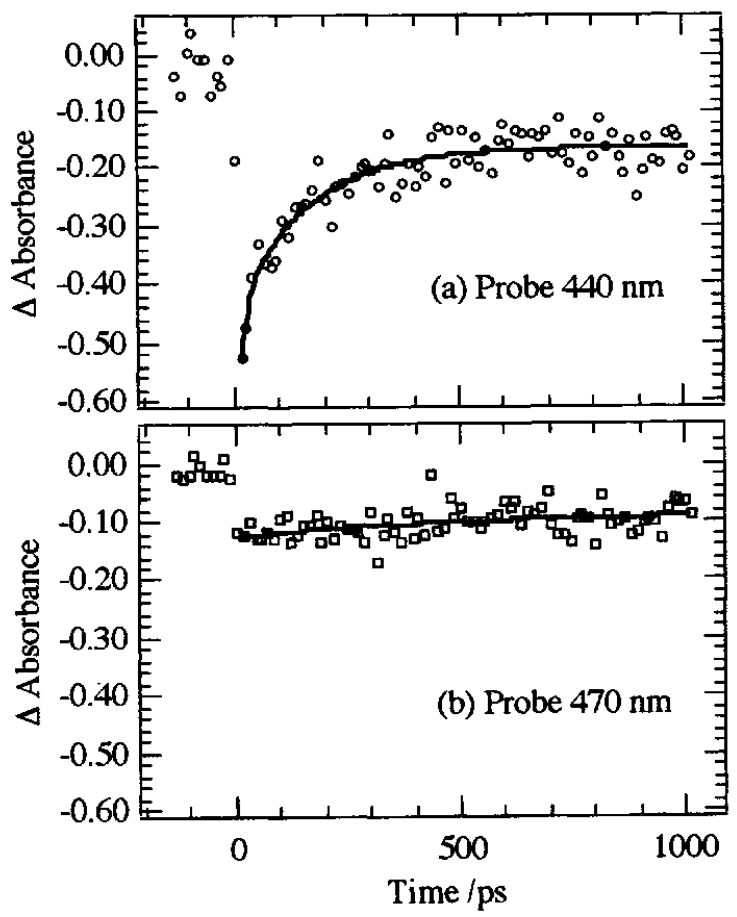

Fig. 5. Time profiles of transient bleaching of $\mathrm{CdS}$ nanoparticles in DTG film at 440 (a) and 470 (b) nm upon excitation with $400 \mathrm{~nm}$ femtosecond pulses. Pump energy $=40 \mu \mathrm{J}$ per pulse. 
due to the quantum size effect in CdS nanoparticles and promotes very fast recombination of electron and hole. In Fig. $5(\mathrm{~b})$, the recovery at $470 \mathrm{~nm}$ shows two component; one with $\tau_{2}=540$ ps $\left(f_{2}=33 \%\right)$ and a very long-lived one ( $\left.\tau_{3}>1 \mathrm{~ns}, f_{3}=67 \%\right)$. The possible origin of the transient species which recover less than $1 \mathrm{~ns}$ is recombination of electrons and holes or excitons via surface states which generated by ionic species in the metrix around the particles. This conclusion is based on X-ray photoelectron spectroscopy which show the existence of cationic nitrogen and anionic chloride ions. These ionic species may act as very stable traps on the particle surface in this self-assembled film. The very long-lived components $(\tau>1 \mathrm{~ns})$ correspond to the radiationless pathways for recombination of the trapped electron and holes in CdS nanoparticles.

There are several possible explanations for the origin of the apparent red-shift of the transient bleaching spectra. One possible explanation is that the transient photobleaching behavior is influenced by positive absorbance contributions from trapped electrons and/or holes. $[4,9]$ It has been suggested that trapped holes show a positive transient absorbance with a peak at around $460 \mathrm{~nm}$.[4,9] However, Fig. 4 clearly indicates that there is no peak in absorbance at $460 \mathrm{~nm}$ upon laser excitation of CdS nanoparticles in the self-assembled films. Thus, the observed transient photobleaching behavior is most likely linked to effects associated with the size distribution of the particles in the film. The time constant of the recovery at $470 \mathrm{~nm}$ is much longer than that at $440 \mathrm{~nm}$. These results can be interpreted as more traps being available on the surface of a large particle (bigger surface area), than on a smaller particle, and hence a longer time is required for the recombination of charge carriers on a larger particle. The possibility of deeper traps being present on the larger particles cannot be excluded but if the traps are generated by ionic species in the matrix interacting with the surface of the particle then it would be unexpected that there would be a difference in the trap energies on the smaller and larger particles.

\section{Conclusion}

Femtosecond bleaching measurement has been used to monitor the electron-hole recombination process of the photoexcited CdS nanoparticles in self-assembled films. A multiexponential fit to the recovery processes of transient absorbance yielded short and long lifetime components. This behavior has been ascribed to the existence of trapped electrons and holes in different energy surface states on the particles. The trap sites have been postulated to be formed by the presence of ionic species in self-assembled film.

\section{Acknowledgments}

We would like to thank Prof. T. Kunitake and Dr. I. Ichinose of Kyushu university for providing DTG samples.

\section{References}

[1] Y. Wang, A. Suna, J. McHugh, E.F. Hilinski, P.A. Lucas and R.D. Johnson, J. Chem. Phys., 92 (1990) 6927.

[2] Y. Wang and N. Herron, J. Phys. Chem., 95 (1991) 525.

[3] H. Inoue, R. Urquhart, T. Nagamura, F. Grieser, H. Sakaguchi, and D. N. Furlong: Colloids and Surfaces A: Physicochem. Eng. Aspects, 126 (1997) 197.

[4] J. Z. Zhang, R. H. O'Niel and T. W. Roberti,: J. Phys. Chem., 98 (1994) 3859.

[5] I. Ichinose, N. Kimizuka, and T. Kunitake, $J$. Phys. Chem. 99 (1995) 3764.

[6] A. Henglein, Chem. Rev., 89 (1989) 1861.

[7] Y. Nosaka, J. Phys. Chem., 95 (1991) 5058.

[8] V. F. Kamalov, R. Little, S. L. Lognov, and

M. A. El-Sayed, J. Phys. Chem., 100 (1996) 6381.

[9] P. V. Kamat, Chem. Rev., 93 (1993) 267 